PROCEEDINGS OF THE

AMERICAN MATHEMATICAL SOCIETY

Volume 139, Number 3, March 2011, Pages 1091-1093

S 0002-9939(2010)10553-1

Article electronically published on August 23, 2010

\title{
ON A QUESTION OF D. SHLYAKHTENKO
}

\author{
IONUT CHIFAN AND ADRIAN IOANA
}

(Communicated by Marius Junge)

\begin{abstract}
In this short paper we construct two countable, infinite conjugacy class (ICC) groups which admit free, ergodic, probability measure-preserving orbit equivalent actions but whose group von Neumann algebras are not (stably) isomorphic.
\end{abstract}

\section{INTRODUCTION}

Two countable, discrete groups $\Gamma$ and $\Lambda$ are orbit equivalent if they admit free, probability measure-preserving actions which generate isomorphic equivalence relations. They are $W^{*}$-equivalent (or von Neumann equivalent) if their group von Neumann algebras are isomorphic. D. Shlyaktenko noticed that, for all known examples of orbit equivalent groups, their group von Neumann algebras are isomorphic and speculated that this might be the case in general. Subsequently, a number of people have also asked the question of whether orbit equivalence of groups implies von Neumann equivalence $([4,[8])$. Motivated by this question, we prove:

Theorem 1. There exist two countable, discrete, infinite conjugacy class groups $\Gamma$ and $\Lambda$ which are orbit equivalent but not $W^{*}$-equivalent. Moreover, the group von Neumann algebras $L \Gamma$ and $L \Lambda$ are not stably isomorphic.

The construction of the groups $\Gamma$ and $\Lambda$ is based on the observation that being an infinite conjugacy class group is not an orbit equivalence invariant. Indeed, from 1 we have that $\Gamma_{0}=S_{\infty}$, the group of finite permutations of $\mathbb{N}$, is orbit equivalent to $\Lambda_{0}=\mathbb{Z}$. This example already shows that there are orbit equivalent groups which are not von Neumann equivalent. Further, notice that the map $\Gamma_{0} \rightarrow \Gamma=$ $\left(\Gamma_{0} \times \mathbb{F}_{2}\right) \star \mathbb{Z}$ turns every pair $\left(\Gamma_{0}, \Lambda_{0}\right)$ of orbit equivalent groups into a pair $(\Gamma, \Lambda)$ of orbit equivalent, infinite conjugacy class groups. Finally, by applying the Kurosh type results for free product von Neumann algebras from [5] we derive that the group von Neumann algebras of $\Gamma$ and $\Lambda$ are not stably isomorphic.

\section{PROOF OF THEOREM}

Before proving the theorem, we recall the notion of stable isomorphism of $\mathrm{II}_{1}$ factors. For a $\mathrm{II}_{1}$ factor $M$ and $0<t \in \mathbb{R}$, the amplification $M^{t}$ is defined as the isomorphism class of $p\left(\mathbb{M}_{n}(\mathbb{C}) \otimes M\right) p$, where $n>t$ is an integer and $p \in \mathbb{M}_{n}(\mathbb{C}) \otimes M$ is a projection of trace $\frac{t}{n}$. It is well known that this isomorphism class does not

Received by the editors September 3, 2009 and, in revised form, April 5, 2010.

2010 Mathematics Subject Classification. Primary 46L10; Secondary 37A20.

Key words and phrases. von Neumann algebra, $W^{*}$-equivalence, orbit equivalence.

(c) 2010 American Mathematical Society Reverts to public domain 28 years from publication 
depend on the choices of $n$ and $p$. Then two $\mathrm{II}_{1}$ factors are called stably isomorphic if one of them is isomorphic with an amplification of the other.

Proof. Let $\Gamma_{0}$ and $\Lambda_{0}$ be two infinite amenable groups and assume that $\Gamma_{0}$ is infinite conjugacy class (ICC) while $\Lambda_{0}$ is abelian. By [3], $\Gamma_{0}$ and $\Lambda_{0}$ are orbit equivalent. Further, by Section 2.2 in [2], the ICC groups $\Gamma=\left(\Gamma_{0} \times \mathbb{F}_{2}\right) \star \mathbb{Z}$ and $\Lambda=\left(\Lambda_{0} \times \mathbb{F}_{2}\right) \star \mathbb{Z}$ are orbit equivalent. We claim that the group von Neumann algebras $M=L \Gamma$ and $N=L \Lambda$ are not stably isomorphic. Let $M_{0}=L \Gamma_{0}$ and $N_{0}=L \Lambda_{0}$ and note that $M=\left(M_{0} \bar{\otimes} L \mathbb{F}_{2}\right) \star L \mathbb{Z}$ and $N=\left(N_{0} \bar{\otimes} L \mathbb{F}_{2}\right) \star L \mathbb{Z}$.

We suppose by contradiction that this is not the case. Therefore we can find an isomorphism $\theta: M^{t} \rightarrow N$, for some $t>0$. Since $M_{0}$ is a factor we can view $M_{0}^{t}$ as a subfactor of $M^{t}$. The commutant of $M_{0}^{t}$ in $M^{t}$ is then equal to $L \mathbb{F}_{2}$. Since the latter is a noninjective factor, by the first part of the proof of Theorem 3.3 in [5] we deduce that a corner of $\theta\left(M_{0}^{t}\right)$ can be embedded into $N_{0} \bar{\otimes} L \mathbb{F}_{2}$ inside $N$ in the sense of Popa (Theorem 2.1 in [7]).

This amounts to the existence of two nonzero projections $q_{1} \in M_{0}^{t}, p \in N_{0} \bar{\otimes} L \mathbb{F}_{2}$, a nonzero partial isometry $v \in N$ and a unital injective homomorphism $\phi: \theta\left(q_{1} M_{0}^{t} q_{1}\right)$ $\rightarrow p\left(N_{0} \bar{\otimes} L \mathbb{F}_{2}\right) p$ such that $x v=v \phi(x)$ for every $x \in \theta\left(q_{1} M_{0}^{t} q_{1}\right)$. Moreover, $v$ satisfies $v v^{*} \in \theta\left(q_{1} M_{0}^{t} q_{1}\right)^{\prime} \cap \theta\left(q_{1}\right) N \theta\left(q_{1}\right)$ and $v^{*} v \in \phi\left(\theta\left(q_{1} M_{0}^{t} q_{1}\right)\right)^{\prime} \cap p N p$ and therefore [6] together with the factoriality of $M_{0}$ implies that $v v^{*} \in \theta\left(\left(\mathbb{C} q_{1}\right) \bar{\otimes} L \mathbb{F}_{2}\right)$ and $v^{*} v \in$ $p\left(N_{0} \bar{\otimes} L \mathbb{F}_{2}\right) p$. Hence $v v^{*}=\theta\left(q_{1} \otimes q_{2}\right)$ for some nonzero projection $q_{2} \in L \mathbb{F}_{2}$, and let $u \in N$ be a unitary such that $\theta\left(q_{1} \otimes q_{2}\right) u=v$. By combining these relations with $x v=v \phi(x)$ we obtain that

$u^{*} \theta\left(\left(q_{1} \otimes q_{2}\right)\left(M_{0}^{t} \bar{\otimes}(\mathbb{C} 1)\right)\left(q_{1} \otimes q_{2}\right)\right) u=v^{*} \theta\left(M_{0}^{t}\right) v=\left(v^{*} v\right) \phi\left(\theta\left(q_{1} M_{0}^{t} q_{1}\right)\right) \subset N_{0} \bar{\otimes} L \mathbb{F}_{2}$.

By [6] this further implies that $u^{*} \theta\left(\left(q_{1} \otimes q_{2}\right)\left(M_{0} \bar{\otimes} L \mathbb{F}_{2}\right)^{t}\left(q_{1} \otimes q_{2}\right)\right) u \subset N_{0} \bar{\otimes} L \mathbb{F}_{2}$. Replacing $\operatorname{Ad}\left(u^{*}\right) \circ \theta$ with $\theta$ and then denoting by $q=q_{1} \otimes q_{2} \in M_{0} \otimes L \mathbb{F}_{2}$ and $r=\theta(q) \in N \bar{\otimes} L \mathbb{F}_{2}$ we can therefore assume that $\theta\left(q\left(M_{0} \bar{\otimes} L \mathbb{F}_{2}\right)^{t} q\right) \subset r\left(N_{0} \bar{\otimes} L \mathbb{F}_{2}\right) r$.

Since the center, $\mathcal{Z}$, of $r\left(N_{0} \bar{\otimes} L \mathbb{F}_{2}\right) r$ is diffuse and $\theta$ is an isomorphism, we derive that $\theta^{-1}(\mathcal{Z})$ is also a diffuse subalgebra which is contained in the relative commutant of $q\left(M_{0} \bar{\otimes} L \mathbb{F}_{2}\right)^{t} q$ in $q M^{t} q$. On the other hand, by [6], the relative commutant of $q\left(M_{0} \bar{\otimes} L \mathbb{F}_{2}\right)^{t} q$ in $q M^{t} q$ is equal to the center of $q\left(M_{0} \bar{\otimes} L \mathbb{F}_{2}\right)^{t} q$, which consists only in the scalars because $M_{0}$ is a factor. Therefore we have reached a contradiction.

We would like to end this note by mentioning the following open question: Are there any examples of $W^{*}$-equivalent groups which are not orbit equivalent?

\section{ACKNOWLEDGMENTS}

The authors are grateful to Professors Sorin Popa and Yehuda Shalom for useful discussions and encouragement.

\section{REFERENCES}

[1] H.A. Dye, On groups of measure preserving transformations. II, Amer. J. Math. 85 (1963), 551-576. MR0158048 (28:1275)

[2] D. Gaboriau, Examples of groups that are measure equivalent to the free group, Ergodic Theory and Dynam. Systems 25(6) (2005), 1809-1827. MR2183295 (2006i:22024)

[3] D. Ornstein and B. Weiss, Ergodic theory of the amenable groups. I. The Rokhlin Lemma, Bull. Amer. Math. Soc. (N.S.) 2 (1980), 161-164. MR $551753(80 \mathrm{j}: 28031)$

[4] N. Ozawa, Amenable actions and applications, International Congress of Mathematicians, vol. II, Eur. Math. Soc., Zürich, 2006, pp. 1563-1580. MR2275659 (2008k:46185) 
[5] N. Ozawa, A Kurosh-type theorem for type $\mathrm{II}_{1}$ factors, Int. Math. Res. Not. (2006), Art. ID 97560, 21 pp. MR2211141 (2006m:46078)

[6] S. Popa, Orthogonal pairs of *-subalgebras in finite von Neumann algebras, J. Operator Theory 9(2) (1983), 253-268. MR703810 (84h:46077)

[7] S. Popa, Strong rigidity for $\mathrm{II}_{1}$ factors arising from malleable actions of w-rigid groups. I, Invent. Math. 165(2) (2006), 369-408. MR2231961 (2007f:46058)

[8] S. Popa, Revisiting some problems in $W^{*}$-rigidity, 2009. Available at http://www.math. ucla.edu/ popa/tamu0809rev.pdf

Department of Mathematics, Vanderbilt University, Nashville, Tennessee 37240 and - Institute of Mathematics "Simion Stollow" of the Romanian Academy, Bucharest, ROMANIA

E-mail address: ionut.chifan@vanderbilt.edu

Department of Mathematics, University of California los Angeles, Los Angeles, California 90095 - and - Institute of Mathematics "Simion Stollow" of the Romanian Academy, Bucharest, Romania

E-mail address: aioana@caltech.edu 